\title{
Hypothesis
}

\section{'Biological' depression; because sleep fails?}

\author{
A.H. Crisp \\ St George's Hospital Medical School, Cranmer Terrace, London SW17 0RE, UK.
}

\section{Introduction}

The melancholy mind has been recognized throughout recorded history. It was given a medical context by the ancient Greeks who claimed for it a relationship with constitution in the form of physique, body chemistry and temperament. It was subject to scholarly analysis by Burton (1927) over three hundred years ago. The disorder has not always been medicalized and some cultures have tended instead to recognize it as a proper and sometimes creative state of mind. It was through the English translation of Kraepelin's writings (1921) that the word 'depression' entered the medical arena. Subsequently Meyer (1934) emphasized the notion that depression was a psychobiological reaction to adverse events. This view has found more recent expression in the writings of Schmale \& Engel (1975) in particular.

In this country the debate concerning the nature and sub-classification of depressive illnesses has become a major preoccupation of psychiatry. The longstanding concept of 'endogenous depression' is handicapped by implying aetiological understanding to what is essentially a phenomenological approach. Protagonists of the various views have sometimes seemingly forgotten that in an analysis of such data you only get out what you have put in in terms of patient groups and symptoms. For instance, the large group of depressed people lurking in medical wards and out-patient clinics has seldom been incorporated into studies of other patients attending hospitals and labelled with the diagnosis. Nor has the rich range of feeling states and their disturbances been incorporated into the symptomatology. These have usually been excluded in favour of apparently hard data such as the presence or otherwise of psychotic phenomena, appetite and weight and sleep changes, all of which can in fact be difficult and elusive features in terms of meaning and

Correspondence: Professor A.H. Crisp, M.D., D.Sc., F.R.C. Psych.

Based on: The Seventh Shorvon Memorial Lecture delivered at The National Hospital, Queen's Square, London, W.C.1. on Thursday, 24 January 1985.

Accepted: 19 September 1985 measurement. To tap this wide spectrum of potentially relevant feeling states and moods one only has to read Burton or Roget's Thesaurus (1953). Paykel (1983) has expressed a measured view about our present state of knowledge in respect of phenomenology and classification. Not much more can be said than that a previous history of a manic episode is probably important prognostically; the presence of anxiety is more common in moderate depressive illness whilst agitation is more common in severe depression. Furthermore, these categorizations may have therapeutic relevance. Meanwhile, there is now a new, elaborate classification DSM III (1980), based strictly on observation and description.

Why has the word 'depression' prevailed for so long? As a word borrowed from physics it seems liable to obscure rather than clarify the nature of the states of mind in question, perhaps bringing to them a spurious sense of understanding in physical terms. In medicine in connection with brain function we already talk about 'depression of consciousness' and 'CNS depressant drugs' and so it could be muddling also to apply the word to disordered mood. If, however, one turns to the Concise Oxford Dictionary then the definitions there seem possibly to be of some relevance. Depression is a lowering or a sinking, a reduction in vigour, especially of trade, the centre of minimum atmospheric pressure and so on, whilst to depress means to lower, to reduce the activity of or to push down or to pull down. These last two alternative ways of looking at the concept are perhaps amongst the most arresting although some of the other definitions invite psychological parallels. Could the word, for instance, all these years have embodied this compelling and pervasive idea that, in a mentally depressing process, both push and pull can be involved? Could the push derive from avoidance of painful experience and the pull from the potential rewards of helplessness and fulfilled dependency needs? Could the depressed state, or for that matter the long ignored concept of inertia described by Freud and amplified by Schur (1970), comprise either one such process or both processes facilitating each other? 
Furthermore, if the variety of distorted moods such as anger, envy, discontent, hatred, sadness, despair, hopelessness, anxiety, that Burton describes and which Engel (1962) attempted to identify as discrete psychobiological entities, are considered as part of and operating within depressed states, then one has a recipe for great complexity and variation within these syndromes. This would be so without taking into account their further distortion when displayed, described or expressed within the doctor/patient relationship and the context of personality. Some of these psychobiologically rooted mood states will contain important elements that are incompatible with each other, for instance when anxiety and anger co-exist. Some will contribute predominantly to vectors which are part of the depressing mechanism whether of the push or pull kind. Others will reinforce vectors generating engagement with the outside world and associated affective reactivity.

\section{Sleep patterns and depression}

The model which I wish to endorse posits man as a prospecting, problem-solving organism with long established biological goals and more recently

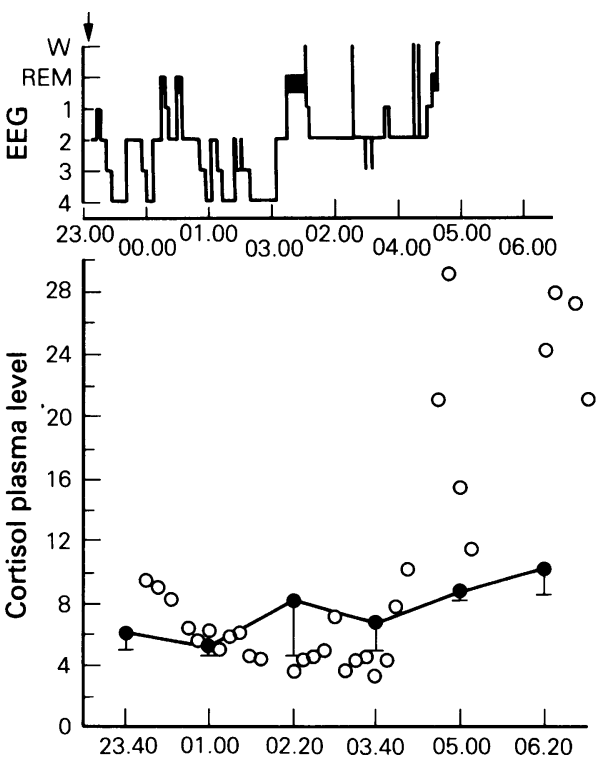

Figure 1 Some typical sleep characteristics of anorexia nervosa in an 18 year old female weighing $32.8 \mathrm{~kg}$, height 1.58 metres. Light, restless sleep with early final waking is evident in the EEG. This feature coincides with, and to some extent is even just preceded by, the beginnings of a massive increase of plasma cortisol levels $(O)$, way above peak normal levels $(-1$, mean and standard deviation of plasma cortisol levels in ten normal young adult females). acquired complex social needs and goals, some of an existential kind. First, let us look at early morning wakening which has for long been regarded as an important marker for severe depressive illness. Early morning wakening within severe depression is a salient complaint, often a bitter one. Many authorities regard it as a biological marker for depression, along with diurnal variation of mood, retardation and/or agitation; these latter disturbances being greatest in the morning. In this sense the phenomenon has often been contrasted with sleeping difficulties in the first half of the night, which tend to be more common in anxious subjects and which are often regarded as more likely to characterize mild depression and neurotic disorder. Certainly, it is easier for most of us to readily identify with initial insomnia and to consider it plausibly related to such feeling states as I have mentioned. But can waking early also be related to either normal mental states or else mental states not qualitatively different from the normal? As we get older we waken earlier (Tune, 1968). Energetic, able and well organized people sleep less and waken earlier than their counterparts (Hartmann et al., 1972) and perhaps we are all capable of this when at our most effective. Some people claim to be able to waken early if needs be, as if some internal alarm clock has been set the evening before. If our mind is active when we go to bed we may not only find it remaining so for the first hour or two thereafter but we may waken with the same thoughts during the night and perhaps also awaken earlier the next day with the same association. Business has never really ceased. Perhaps such mechanisms also operate within psychiatric illness including severe depression.

\section{Sleep studies}

My own line of enquiry into early morning wakening initially appeared remote from depressive illness since it was concerned with anorexia nervosa. Such people, who are usually young and hence come from a population which sleeps well on the whole, often waken early in the morning but deny it (Crisp, 1967).

The patient in Figure 1 with severe anorexia nervosa has much reduced 'polygraphic' sleep. She wakens finally just before 05.00 hours after under six hours of total sleep, much of it light. Prior to wakening a major surge in her plasma cortisol level begins as part of the intense arousal about to overtake her. It was evident that the clinical features associated with such sleep patterns were nutritional rather than affective in kind. The more underweight and starved the individual the greater the sleep disturbance irrespective of mood. Perhaps we were witnessing the breakup of the contrived eight hour or so long package of sleep induced in us in early life in temperate climates by our parents, themselves seeking uninterrupted sleep, and 
despite our own relatively unrequited hunger at the time. Most of us learn to do without this meal during the night even though, presumably, this step may distort our feeding patterns during the rest of the day. However, without nourishment to the extent found in anorexia nervosa there is little point in lengthy sleep. Some initial corrective to falling body temperature such as sleep may be necessary but thereafter it is more important, biologically, to waken, rise and forage. This erosion of sleep is only one aspect of the arousal experienced by the anorectic, the other being the high level of activity characteristically sustained during the day. Such intense arousal, eroding sleep and heightening restlessness during wakefulness together with the associated plasma cortisol changes (presumably escaping dexamethasone suppression because of their high surge) it was thought might have implications for other conditions (vide infra).

Our next series of experiments also involved studies of sleep and mood in anorectics as they gained weight to normal levels and obese subjects as they lost substantial amounts of weight. In the former group normal sleep was reconstituted whilst in the latter group it became eroded. These changes arose unrelated to changes in mood and appeared to be dependent upon weight change. It seemed possibly also to be a matter of available calories. Concurrently, we (Crisp \& Stonehill, 1973, 1976) tested the hypothesis that sleep disturbances in a variety of psychiatric disorders would be found to be related to weight changes irrespective of diagnosis. Thus weight loss would be associated with reduced total sleep time, increased interruption of sleep and earlier wakening whilst weight gain would be associated with the converse. We were aware, of course that such weight changes not infrequently occur in psychiatric illness, especially, it is said, depressive illness. Under these circumstances doctors duly note the weight change, perhaps most often weight loss, as indicative of the severity of the depressive disorder confronting them but we do not often seem to stop and think of its biological consequences.

Most of us have great difficulty in losing weight. For the most part we maintain a steady state and this self regulation of shape and weight seems to be governed by both biological and social factors. Social and psychological factors include the reactions of those around us, our appearance in the mirror, the tightness of our clothes and how we feel about our bodies and about being in them. Biological factors are ill understood. They may include a central mechanism governing calorie intake. For instance, our own group (Phillips et al., 1975) has shown that high carbohydrate intake one day is associated with increased rapid eye movement (REM) sleep during the subsequent night whilst Seigel (1975) has shown that amount of REM sleep in small mammals is inversely related to the next day's carbohydrate intake. Any such regulatory mechanism, discernible within the architecture of sleep, could supposedly operate either directly through, say, blood glucose levels or perhaps indirectly through plasma free and bound tryptophan levels or tryptophan/tyrosine ratios according to the Wurtmann \& Fernstrom (1975) hypothesis. Tryptophan is a precursor of serotonin (5HT) which itself is thought possibly to mediate REM sleep (see Crisp, 1980). We should remember that the brain probably processes all kinds of information and perhaps can get overloaded with nutritional information just as much as with 'social' information and perhaps can even get 'depressed' (the inertia of satiation) as one stance open to it in relation to its regulation of food intake. It seems likely then that the architecture of sleep, and perhaps also its experience, is sensitive to aspects of daily diet as well as to the impact of sustained starvation and weight loss. It would certainly seem that any such biological regulatory mechanisms may be over-ridden and even severely distorted at times by the kind of 'depression' under consideration here together with its contributory and corporate affects, the disturbances of which may have social origins. The major impact which consequent weight loss might then have in biological terms - its impact on the body's chemistry and its behaviour - has been largely ignored within psychiatric syndromes.

In our study of psychiatrically ill patients therefore we developed standardized questionnaires and assessed aspects of sleep, weight and mood and changes in them in a series of 375 new psychiatric out-patient referrals, examining these phenomena in relation to the various psychiatric diagnoses. Some of our principal findings relevant to the present matter were as follows:

A history of obesity confers a propensity for major weight change (increase or decrease) during the illness whatever its nature and has no specific association with a current diagnosis of endogenous depression or manic depressive psychosis. A history of having once been thin is associated with a current diagnosis of neurotic depression and/or very disturbed mood especially tension. A history of weight loss during present illness is more likely to have arisen in neurotic depression and anxiety state than in endogenous depression.

A present state of thinness is more likely amongst those with the diagnosis of personality disorder and who are tense/angry rather than those who are anxious/sad. Most broken sleep and less total sleep characterizes those who are angry. A population of psychiatrically ill people overall display a tendency to report having not changed their time of going to bed or time of waking but to take longer to go to sleep, have more broken sleep and less total sleep than before the illness. Moreover, time of going to bed and falling 
asleep is earlier in endogenous depression than in other diagnoses. Time of waking is early in endogenous depression and late in anxiety state compared with the total population. Total sleep is less amongst patients with neurotic depression than the other diagnoses, and greatest in those having endogenous depression. The sleep period in endogenous depression occurs earlier within the 24-hour cycle than is the case with the overall population and this contrasts with the later sleep periods of subjects with neurotic depression and anxiety state.

Finally, within the total population and irrespective of diagnosis, there is an important association, especially in relation to the previous few weeks, between changes in weight and changes in sleep. Weight loss is associated with a reduction of total sleep, compounded of more broken sleep and earlier waking. Weight gain is associated with an increase in total sleep compounded of less broken sleep and later waking (Figure 2).

The conclusion from our study in respect of so called 'endogenous' depression was that people with such severe depression do not necessarily waken all that much earlier than before their illness. Moreover, they sleep for quite a long while. Firstly, they go to bed earlier and go to sleep earlier than others so it would not be surprising if they wakened earlier. Thus it would seem that the hallmark of severe depression is, in fact, often not so much reduced sleep in the second half of the night but complaint about waking too early.

\section{Discussion and hypothesis}

Why do severely depressed people complain about waking early when patients with anorexia nervosa minimize it? Many normal people waken quite early and are delighted so to do. People with severe depression waken to find they are overwhelmed by gloom and despair. They have no solutions to the problems surrounding them and from which they may believe themselves to have been protected during sleep. Therefore, they complain of wakening early and often seek further oblivion in the form of CNS depressant drugs. It is this diurnal variation of mood in which despair and other features of the depressive syndrome are worse in the early part of the waking day which seems to me to be one of the real puzzles.

What do we wake from and wake to? Let us revert to the concept of ourselves as problem-confronting, solution-seeking creatures needing to prospect through the day in our search for food and security and its trappings. Oswald (1970) proposed that REM sleep in particular might reflect CNS updating and reprogramming processes related to such sociobiological purposes, preparing us thereby for this task each day. (1) The individual can then take the best possible action, given his/her basic coping capacities, and the external and internal (personal) contexts of the problem. I emphasize the word action at this point sothat I can later set it conceptually against the inaction that characterizes us in severe depression. (2) Depression, in evolutionary terms, may be nature's way of

Figure 2 Sleep patterns (diagrammatic) related to diagnostic category and mood state within a total psychiatric population $(n=375)$ (Crisp \& Stonehill, 1973, 1976). Measurements by standardized self-reports and interviewers' ratings. Note the sleep characteristics of the main population, divided into those who had gained weight compared with those who had lost weight irrespective of diagnosis. Patients labelled 'endogenous depression' did not, as a group, display reduced total sleep time. Slightly earlier waking was related to earlier sleep onset following earlier retirement to bed: ( $\square$ ), in bed awake; $(\square)$ in bed asleep. 
discarding those who, in later life, are unable to continue to adapt. Equally, such a challenge in terms of survival may provide the pivot for real change so long as there is inner potential and complementary assistance from without. Meanwhile the REM process is not conceived of as simply one of message processing. It is perhaps also one of problem-solving at these times when the system is especially capable of modification through learning.

REM sleep is a cerebrally active state. Cerebral blood flow is high at this time. There is special activity in the autonomic nervous system, including for instance such 'instinctual' activity as penile erection, perhaps as part of the reprogramming exercise. Meanwhile, the musculo-skeletal system, the rest of the body, is disengaged, the muscles are flaccid. During REM sleep especially it seems that we dream. Dreaming may reflect this process within which the memory stores are mobilized in their coded form thus enabling them to be more receptive to the latest information input both in terms of processing it and being modified by it. Freud, with his conviction of the basic relevance, for human experience, of the clash between biological and social needs, claimed that dreaming was a prime arena for this encounter. Furthermore, he claimed that memories, not usually accessible to awareness during the day, seemed to arise during the night and could then be accessed with certain techniques. It was Jung who considered that these memories were not only those of the individual himself or herself but were also the genetically transmitted memories of evolutionary time. Presumably the latest information is necessary for the organism to cope best with the next day's task. However, it must be set against the enormous background of previous experience. Thus dreams, as many have claimed, may be as close as we come to prophesy - on waking, the future, good or bad, is perceived most starkly.

Supposing the information coming into the system is presenting insoluble problems to the individual, yet the information is important. Initial responses may have included the experience of such feelings as anxiety or anger. Grappling with this task of assimilating such information with a view to effective action and fulfilment is ultimately beyond the system and the outcome is defeat. The individual wakens in the morning with no problems solved and with no new resources to tackle the tasks which were overwhelming the previous day. Lewin (1950) described the 'blank dreams' which, as a psychoanalyst, he recognized as the harbingers of depression in his patients with cyclical affective illness. Inaction prevails. The reduced REM latency claimed by some as a feature of people with depression could, if it exists, perhaps reflect an attempt by the organism to embark afresh each night on the necessary tasks that I have just described, given the previous failure. The possible relationship of this to the phenomenon described earlier of the severely depressed person retiring to bed and going off to sleep earlier than before remains unclear. Retirement to bed may simply be another facet of the need to avoid the pain of wakefulness.

If, within sleep, no solution can be found to a problem whether related to realistic or unrealistic goals, then it might seem natural for inertia, retardation, loss of drive, loss of libido, to result. The problem-solving system has ground to a halt and with it the drive which is normally reprogrammed regularly within REM sleep. This would not perhaps be all that different from the protective conservation-withdrawal stance. Alternatively, or in association, there might also be agitation or agitated stupor if anxiety and arousal is still being generated within the system. Gloomy, non-productive thoughts then become the substance of the next night's task and the state of mind is perpetuated. During the day there is some stimulation which tends to distract the individual but this may be insufficient to break the cycle. Then, during sleep, orientation to the outside world is reduced to memory once more. Orientation is now, instead, exclusively towards the self and preparation for the morrow. Irrelevant input from the previous day is discarded and the problem that won't go away and cannot be solved again prevails. Once again it proves overwhelming and on waking the individual is again inert there is no direction in which he can go. Neither flight nor fight, manipulation nor negotiation is possible. Such inertia as a major feature each morning will attract our label of severe 'depression' and indeed may then be cardinal evidence of a need for re-evaluation, for instance, of relationships, and a redirection of purpose for the individual. Inability to achieve this may prove a fatal flaw.

The relationship between this model of depression and the known effects of certain treatments invites consideration. For instance if the individual is treated by sleep deprivation, either selectively or totally, and in order to achieve this there is a background of social stimulation throughout the night (e.g. 'occupational' therapy, playing games) then one would expect some remission of symptoms throughout the next day as found by Elsenga \& van den Hoofdakker (1982/83) but the effect would not extend beyond that. Treatments that did not allow or even blocked the need for problem-solving would sooner or later be counterproductive. However, there could be advantages for appropriate combinations of treatments capable of reengaging or enhancing problem-solving resources. It has been argued (Crisp, 1980) that some tricyclic 'antidepressant' drugs work by rekindling drive behaviour, perhaps at least in part through their impact on REM sleep which is not so much reduced as modified. Thus their effect would be to re-engage the person with the outside world and hence increase the 
opportunities of new and potentially therapeutic stimulation. Meanwhile, the basic problem-solving task in which the individual has failed can be taken up again within psychotherapy. Such psychotherapy could be both cognitive/behavioural as advocated by Beck (1976) and relationship oriented as advocated by Jacobson (1975), these processes facilitating each other.

The model also invites speculation as to how electroconvulsive therapy (ECT) works. Perhaps we should ask the question 'Is there some biological purpose of epilepsy, which is inherent in us all?' Perhaps naturally and subliminally it reintegrates central nervous system functions when disorganization has arisen or is threatening in response to intolerable arousal. Some epilepsy is perhaps restorative! If induced epilepsy was to operate similarly then it might best do so in those depressed subjects whose mental paralysis was the end product of overwhelming stimulation. High arousal is not always evident in depressive disorder itself but may potentially be there, and in my clinical experience this is often so if, for instance, the depressed individual is confronted with his psychological task. Hill (1954), whilst reviewing theories of mode of action of ECT suggested that it might work by reinforcing denial. In naturally occurring epilepsy there is memory loss as with ECT. It seems to have been shown that memory loss as such is not the mechanism whereby ECT works. Nevertheless, I would think that disruption of recent memory processes in the sense that $I$ have been talking about may be an important aspect of the therapeutic effect of

\section{References}

BECK, A.T. (1976). In Cognitive Theory and the Emotional Disorders. International University Press: New York.

BURTON, R. (1927). In The Anatomy of Melancholy, all English text, Dell, F. and Jordan-Smith, P. (eds). Tudor Publishing Co.: New York.

CRISP, A.H. (1967). The possible significance of some behavioural correlates of weight and carbohydrate intake. Journal of Psychosomatic Research, 11, 117.

CRISP, A.H. (1980). In Anorexia Nervosa: Let $\mathrm{Me} B e$. Academic Press: London.

CRISP, A.H. \& STONEHILL, E. (1973). Aspects of the relationship between sleep and nutrition: A study of 375 psychiatric out-patients. British Journal of Psychiatry, 122, 379.

CRISP, A.H. \& STONEHILL, E. (1976). In Sleep, Nutrition and Mood. Wiley: London.

ELSENGA, S. \& VAN DEN HOOFDAKKER, R.H. (1982/83). Clinical effects of sleep deprivation and clomipramine in endogenous depression. Journal of Psychiatric Research, 17, 361 .

ENGEL, G.L. (1962). In Psychological Development in Health and Disease. Saunders: Philadelphia.
ECT. Perhaps the abolition of recent memory is a minor aspect of the mechanism called into play. Perhaps the epileptic fit has an effect also on the links between affect and memory.

It has been argued here that some depressive states can provide the seedbed for personal growth. Some clinicians emphasize the therapeutic importance for patients who have become accustomed to complaining in physical terms or who have developed even more global defences against the experience of 'depression', of being able instead to tolerate and express their underlying affective distress. This step is seen as a necessary enabling one if the individual is to regain awareness of the associated existential problem with the consequent potential to communicate that. The capacity to achieve these steps and then to solve the problem with psychological help will depend on such variables as age, personality resource and the nature of help available. People who can benefit from such an experience may ultimately emerge 'sadder but wiser' and freer from morbid depression. Others may be condemned to chronic or recurrent disability.

So called 'depression' is one of the most common syndromes of our time. It is an unsatisfactory word. I, like others, prefer such words as dysphoria, dysphoric inertia, dysphoric agitation. Such dysphoric states can be related to real problems demanding solutions. If tolerated and confronted, then with help they can sometimes teach us, both individually and as social groups, more about ourselves, thereby enhancing our capacity to survive.
HARTMANN, E., ZWILLING, G.R. \& BAEKELAND, F. (1972). Personality traits and life style data for long and short sleepers. Archives of General Psychiatry, 26, 463.

HILL, J.N.D. (1954). Psychotherapy and the physical methods of treatment in psychiatry. Journal of Mental Science, 100, 360.

KRAEPELIN, E. (1921). In Manic-depressive Insanity and Paranoia. E. and S. Livingstone: Edinburgh.

JACOBSON, E. (1975). The psychoanalytic treatment of depressed patients. In Depression and Human Existence, Anthony, E.J. and Benedek, T. (eds). Little, Brown: Boston.

LEWIN, B.D. (1950). In The Psychoanalysis of Elation. Norton: New York.

MEYER, A. (1934). The psychobiological point of view. In The Problem of Mental Health, Bentley, M. and Cowdry, E.V. (eds). McGraw-Hill: New York.

OSWALD, I. (1970). Sleep the great restorer. New Scientist, 46, 170.

OSWALD, I., BERGER, R.J., EVANS, J.I. \& THACORE, V.R. (1964). Effect of 1-tryptophan upon human sleep. Electroencephalography and Clinical Neurophysiology, 17, 603. 
PAYKEL, E.S. (1983). The classification of depression. British Journal of Clinical Pharmacology, 15, 155.

PHILLIPS, F., CHEN, C.N., CRISP, A.H., KOVAL, J., McGUINNESS, B., KALUCY, R.S., KALUCY, E.C. \& LACEY, J.H. (1975). Isocaloric diet changes and EEG sleep. Lancet, ii, 723.

ROGET'S THESAURUS of English Words and Phrases, revised by Dutch, R.A. (1953). Penguin Books: Harmondsworth, Middx. U.K.

SCHUR, M. (1970). A principle of evolutionary biology for psychoanalysis. Journal of the American Psychoanalytical Association, 18, 442.
SCHMALE, A.H. \& ENGEL, G.L. (1975). The role of conservation-withdrawal in depressive reactions. In Depression and Human Existence, Bentley, E.J. and Benedek, T. (eds). Little, Brown: Boston.

SIEGEL, J.M. (1975). REM sleep predicts subsequent food intake. Physiology and Behaviour, 15, 399.

TUNE, G.S. (1968). Sleep and wakefulness in normal human adults. British Medical Journal, 2, 269.

WURTMANN, R.J. \& FERNSTROM, J.D. (1975). Control of brain monoamine synthesis by diet and plasma amino acids. American Journal of Clinical Nutrition, 28, 638. 